\title{
Requirements of Data Visualisation Tools to Analyse Big Data: A Structured Literature Review
}

\author{
Joy Lowe ${ }^{(\bowtie)}$ (1) and Machdel Matthee (1) \\ Department of Informatics, University of Pretoria, Pretoria, South Africa \\ ul6331878@tuks.co.za, machdel.matthee@up.ac.za
}

\begin{abstract}
The continual growth of big data necessitates efficient ways of analysing these large datasets. Data visualisation and visual analytics has been identified as a key tool in big data analysis because they draw on the human visual and cognitive capabilities to analyse data quickly, intuitively and interactively. However, current visualisation tools and visual analytical systems fall short of providing a seamless user experience and several improvements could be made to current commercially available visualisation tools. By conducting a systematic literature review, requirements of visualisation tools were identified and categorised into six groups: dimensionality reduction, data reduction, scalability and readability, interactivity, fast retrieval of results, and user assistance. The most common themes found in the literature were dimensionality reduction and interactive data exploration.
\end{abstract}

Keywords: Big data visualisation - Visualisation tools $\cdot$ Visual analytics

\section{Introduction}

Big data is growing at an exponential rate due to unprecedented data capture from smart devices, the internet of things, measurement and sensor technology, transaction data, metadata, and social media (Ali et al. 2016; Gisbrecht 2013; Resnyansky 2019). With an estimated 3.7 billion internet users globally (Marr 2018) the internet is a significant factor in the generation of data. Google, the most popular search engine, receives at least 5.5 billion search queries per day (Sullivan 2016) and processes 24 petabytes of data per day (Ali et al. 2016). User generated social media data is another significant contributor to big data, with Facebook reporting 2.32 billion active monthly users (Noyes 2019a) and Twitter reporting 321 million active monthly users with 500 million tweets posted daily (Noyes 2019b)

Big data is defined as large, complex, high velocity data sets (Seokyeon et al. 2015). Big data is characterised by volume, velocity, value, variety, variability, and complexity (Katal et al. 2013). Due to the nature of these large, heterogeneous data sets, interpretation and comprehension of the data is often difficult, and current data storage and analysis technologies are no longer able to effectively store and analyse such large datasets (Genender-Feltheimer 2018). However, using big data brings new opportunities for research and new insights across a wide variety of fields (Resnyansky 
2019) and by visually exploring and analysing the data, unexpected discoveries can be found.

Data visualisation is a solution to aid meaningful analysis, accessibility and interpretation of big data as it relies on human cognitive capabilities to process visual information (Gisbrecht 2013). Visualising data creates an image that the user is able to parse in three subtasks: perceptual grouping, image segmentation and object recognition (Zhu 2003). Image parsing is an efficient means of comprehending large datasets because grouping or clustering of data points, as well as outliers, become apparent. Further, the inclusion of interactive technology and visual analytics allows users to gain more information about specific data points and areas of interest through visual exploration, which facilitates the formation, testing and validation of hypotheses (Elmqvist and Fekete 2010).

Increasingly complex and sophisticated data analytics and visualisation algorithms are required in response to the increasing significance of big data (Gisbrecht 2013). The velocity at which the data is generated produces the need for real time analysis. This paper will explore and compare various techniques which have been developed in response to these requirements to effectively visualise and comprehend big data.

\section{Literature Background}

\subsection{The Growth of Big Data}

Big data has been hailed as one of the greatest challenges of the 21 st century due to the volume of data, the speed at which the data is being generated, as well as the inconsistency of the data (Katal et al. 2013). A number of factors contribute to the growth and complexity of big data including the internet of things (IOT), click stream data, and social media data. Big data has become an integral part of business, social media, scientific research and several other fields (Olshannikova et al. 2015) and the data is predicted to grow even further, at faster rates.

\subsection{The Need for Visualisations to Comprehend Big Data}

Big data is now beyond human comprehension through simple exploration and investigation of the data (Long and Linsen 2011). People are able to comprehend the world more quickly and easily through visual cues, and this extends to visualising data. Vision is the most valuable human sense and most people prefer visual representations to other sensory information.

In order to capitalise on the big data revolution and gain as much insight as possible, it is imperative that the data is analysed efficiently and accurately, and thereafter visualised to allow for the fast interpretation of the data, much of which is generated in real time.

Furthermore, visualisations of data make trends and patterns in the data much more apparent such as clustering, the distribution of the data, and correlation within the data (Long and Linsen 2011). The goal of many businesses regarding data analysis is to 
recognise such patterns, emphasizing the need for data visualisation to achieve strategic objectives.

Visualising data is a means of "uniting the abstract world of data with the physical world through visual representation" (Olshannikova et al. 2015). Visualisations can be likened to the front end of big data (Wang et al. 2015) and can be used to access and interpret the data, making the insights and trends more apparent.

\subsection{The Need for Awareness of Data Visualisation Techniques Among Professionals}

Many business professionals now need to be educated in data related information and become "data literate" in order to generate business reports as well as interpret reports and business intelligence executive dashboards. These business professionals must be made aware of the features and limitations of each visualisation technique and visualization software or tool, particularly with interactive dashboards becoming more popular. In this paper some visualization tools and techniques will be discussed.

\subsection{Commercially Available Business Intelligence and Visualisation Tools}

There are a number of currently available commercial Business Intelligence tools that include the ability to create visualisations, which can be utilized by professionals in a number of different fields and industries. Figure 1 below shows the Magic Quadrant for Analytics and Business Intelligence Platforms published by Gartner in February 2019. This quadrant displays the 'leaders', 'visionaries', 'challengers', and 'niche players' of business intelligence platforms and provides a good overview of the landscape of the industry at present.

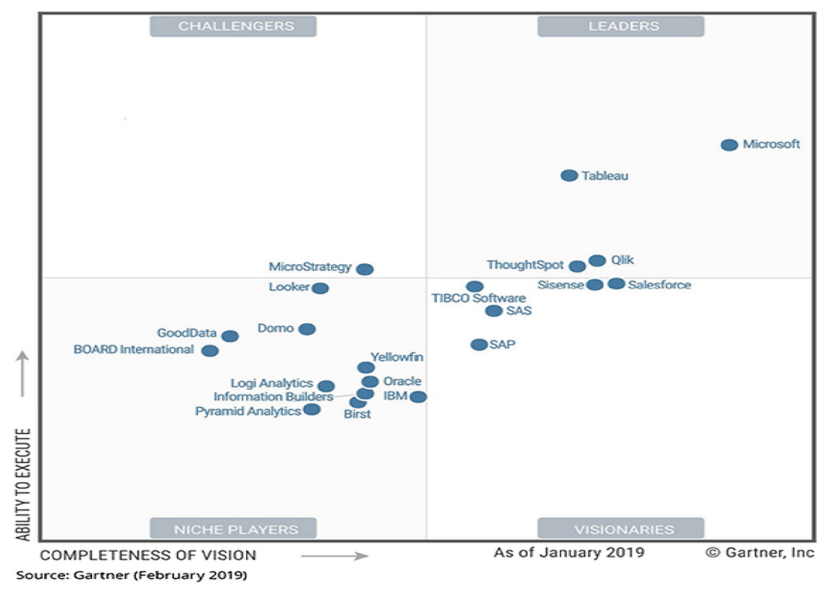

Fig. 1. Gartner magic quadrant for analytics and business intelligence platforms (Orad 2019) 


\section{Research Method}

A systematic literature review was carried out during the course of this research. The following eight steps are suggested when conducting a systematic review of Information Systems research (Okoli and Schabram, n.d.):

1. Identifying the purpose and intended goals of the literature review

2. Outlining the protocol and detailed procedure to be followed

3. Searching for the literature

4. Practical screening/screening for inclusion

5. Quality appraisal/screening for exclusion

6. Data extraction

7. Synthesis/analysis of studies

8. Writing the review.

\subsection{Research Question}

What are the requirements of data visualisation tools used to analyse big data?

\subsection{Search Terms}

Title (data) AND title (visual*) AND abstract (big data).

The wild card search term "visual*" was used to cater for all variations of the words that derive from the common stem 'visual' including visualise, visualising, visualisation, visualisations, as well as being inclusive of both variations of spelling for each of these words: the American spelling which includes a ' $z$ ' and the English spelling which contains an ' $s$ '. In the case where a search engine did not support wild cards, all possible variations of the word were specified using OR Boolean clauses so that any of the spellings would be included in the search results.

\subsection{Selection Criteria}

\section{Inclusion Criteria}

- Articles published between January 2014 and October 2019 were included to ensure the most up to date research was evaluated since the field of big data visualisation is changing rapidly.

- Peer reviewed journal articles and conference papers were included.

- Only articles written in English were included.

\section{Exclusion Criteria}

- Articles published prior to January 2014 and after October 2019 were excluded.

- The following document types were excluded: periodicals, editorials, books, and book chapters. 


\subsection{Source Selection}

The following sources and databases were consulted during the course of this research:

- $\mathrm{ABI} /$ Inform

- ACM Digital Library

- EBSCOhost

- ScienceDirect

The Prisma flow chart below shows the number of articles identified through each database and the process of exclusion that was followed to reach the final 31 articles that were included in the literature review (Fig. 2).

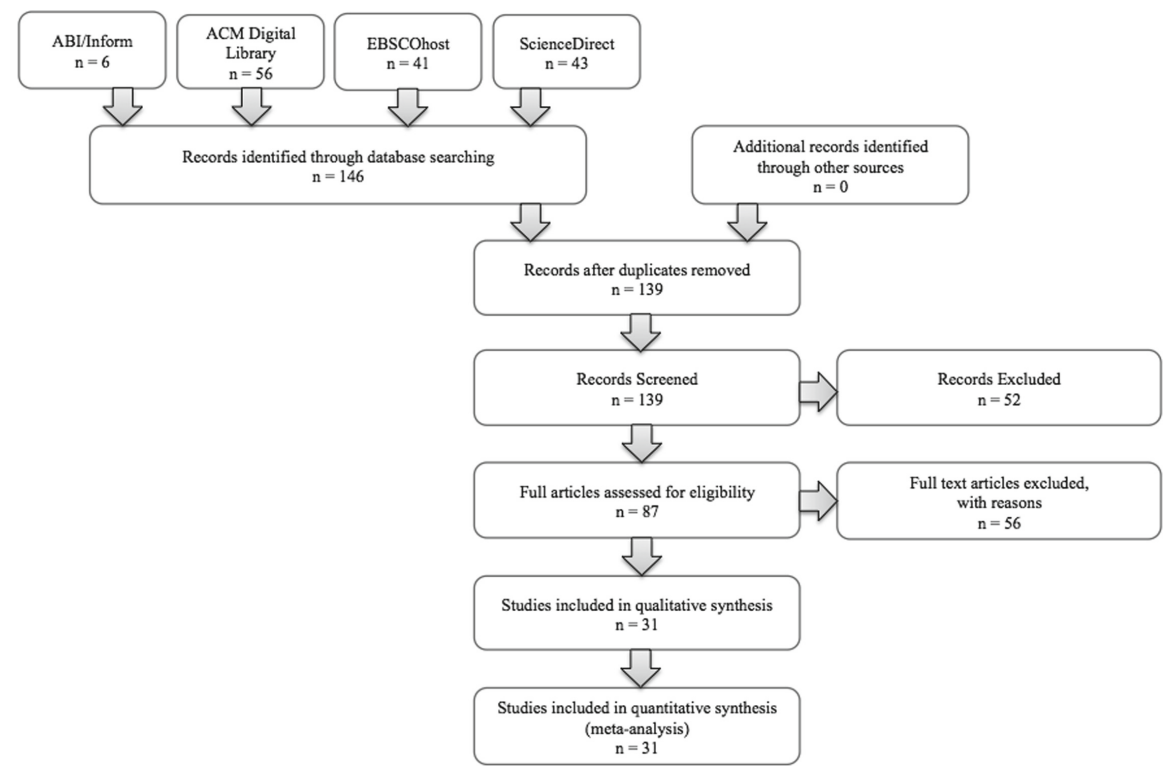

Fig. 2. Prisma flow chart

\section{Analysis of Findings}

After reviewing the literature, many authors are in agreement that data visualisation will become increasingly important as big data becomes more prevalent in more organisations and in order to interpret the data, effective visualisation techniques will be required (Wang and Li 2019). There has also been a call to improve current visualisation techniques ( $\mathrm{Li}$ et al. 2016; Olshannikova et al. 2015) in order to handle the challenges of visualising big data.

A combined list of requirements of data visualisation tools to interpret big data found in the literature are shown in Fig. 3 below. The bar graph shows the number of papers included in the literature which fall into each category. Note that some articles 
address more than one category, thus the total of the values of the bars in Fig. 3 is greater than the total number of articles included in the literature review.

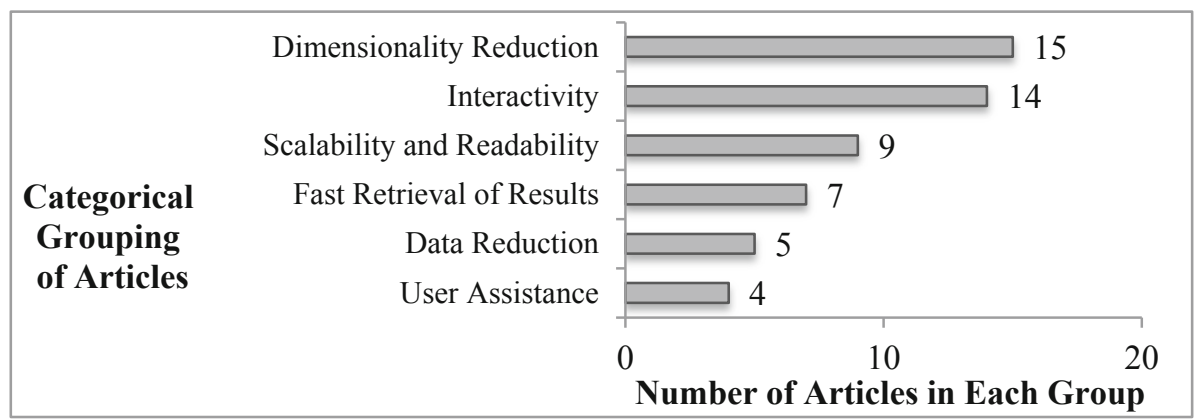

Fig. 3. Number of articles in each category

\subsection{Dimensionality Reduction}

The dimensionality reduction category contributed the highest number of papers (15 of 31). Dimensionality reduction is a key requirement of big data visualisation (Zou et al. 2016) because many of the large datasets that exist today contain multiple dimensions, however, humans can only perceive three dimensions, giving rise to the need for algorithms that can reduce datasets to two or three dimensions which can be visualised. There are a number of statistical algorithms which can achieve this including Principal Component Analysis (PCA), t-distributed stochastic neighbour embedding (t-SNE) and diffusion maps (Agrawal et al. 2015; Fernandez et al. 2015; Genender-Feltheimer 2018; Gisbrecht and Hammer 2015; Shirota et al. 2017). Mapping multidimensional datasets into clusters that are represented in two or three dimensions is also common and allows the partitioning of data into similar groups (Keck et al. 2017). It is useful to maintain the original structures of the data (Fernandez et al. 2015) so that further analysis can be carried out after identifying certain clusters or patterns of interest during the two- or three-dimensional exploration of the data (Keck et al. 2017; Xie et al. 2016). Advantages of dimensional reduction include easily understood visualisations, reduced data quality challenges, and improved computational efficiency (Genender-Feltheimer 2018).

Dimensionality reducing algorithms are either linear, or non-linear. Linear algorithms are only able to learn linear underlying topological spaces, while non-linear algorithms are able to learn complex underlying topological spaces and focus on preserving neighbourhood geometry (Genender-Feltheimer 2018). Dimensionality reduction algorithms can also be grouped into supervised and unsupervised algorithms. Supervised algorithms require a 'training' data set to 'learn' from, and subsequently the model is applied to a second 'scoring' data set. Unsupervised algorithms do not require a training dataset, but rather, are able to detect patterns in the data (Mwangi et al. 2014). Examples of supervised algorithms include Linear Discriminant Analysis and Linear Regression; and examples of unsupervised algorithms include neural networks and 
other deep learning methods. Supervised algorithms have the potential to be tainted with human bias, as the training dataset contains user defined target labels (Mwangi et al. 2014).

\subsection{Interactivity}

By providing a visual interface which the user can interact with, the user is able to intuitively comprehend the data, perceive the underlying patterns (Lugmayr et al. 2017) and query the data visually, without the need for programming knowledge. The ability for a user to interact with a visualisation makes visual exploration of the dataset possible and this has the major advantage of combining both human and machine intelligence (Chen et al. 2019) to uncover unexpected and interesting phenomena within the dataset (Cho et al. 2014). The benefits of visual analytics are "visual perception, interactive exploration, improved understanding, informed steering and intuitive interpretation" (Liu 2019). This has the potential to uncover patterns in the data using a bottom-up approach (Ruan and Zhang 2017) or to test theories and search for evidence within the data using a top-down approach (Genender-Feltheimer 2018; Mwangi et al. 2014). Visualisation tools should enable the user to drill down into areas of interest within the data (Zhao et al. 2017) to aid in the visual analysis.

Several authors write about the need to keep the user informed at each step of the visual analytics process. This gives the user situational awareness and keeps the user informed about the underlying processes being run in order to render the data or return results (Zhao et al. 2017). Currently, many visualisation tools are a "Black Box" and do not give enough feedback to the user. Results are produced without a description of how the results were calculated, and in some visualisation systems, the user is not given the option to select a statistical method of their choice (Seokyeon et al. 2015).

\subsection{Scalability and Readability}

The displays on which the visualisations are rendered have a limited number of pixels, thus the lowest granularity possible is to plot one data point onto one pixel (MolinaSolana et al. 2017; Olshannikova et al. 2015; Yang et al. 2015). Visualisation tools are required to "squeeze a billion records into a million pixels" (Bikakis 2018) emphasizing the need for summarisation of data, particularly when creating visualisations. If the dataset is not sufficiently summarised, or if every data point is plotted, this may result in 'over plotting' which is inefficient and it can be difficult to discern the patterns and trends within the data ( $\mathrm{Li}$ et al. 2016). The number of pixels is even lower on mobile device screens, thus the scalability of visualisations for mobile devices are even more important (Li et al. 2016; Molina-Solana et al. 2017). Furthermore, attempting to plot all data points overloads the users' cognitive capabilities, thus it is important to ensure readability and scalability across all screen sizes and in all contexts (Eldawy et al. 2015). In order to achieve this, visualisation tools should include zooming; overview and detail; and brushing of the visualisation (Li et al. 2016). The visualisation tools should also use the approach of providing a high level overview of the data initially, and subsequently load more detailed data as the user queries and drills down into areas of interest (Agrawal et al. 2015). 


\subsection{Fast Retrieval of Results}

It is imperative that data visualisation and visual analytics systems retrieve data fast enough so that the user does not lose their focus and momentum when visually analysing a dataset. The intention is to hold the users concentration, enabling them to problem solve at a high level and creatively formulate queries to investigate further (Liu 2019). Several authors agree that the result should be returned within one second, while some authors specify not more than ten seconds (Li et al. 2016).

One approach to address this is called approximate query processing, whereby an approximation of the data is returned quickly and the exact results are continuously processed in the background while the user moves onto other queries (Zhao et al. 2017). When the query has finished processing, which may take up to ten minutes, the user is informed and the discrepancies between the original estimate and the final results are displayed to the user (Moritz et al. 2017). Generally, it is more important to get a broad overview of the data, rather than exact figures, and in this regard, the approximate processing approach works well. Displaying the confidence interval of the estimate during the initial load of the data gives further benefit to this method, as the user is given an estimate and an indication of how accurate the estimate is. This is particularly useful when the results of simple queries are needed urgently (Masiane 2019; Zhao et al. 2017).

Other approaches which may speed up data retrieval include catching or prefetching the results of frequent queries. It may also be advantageous to divide the data processing across several computers. Online Analytical Processing (OLAP) is another method which speeds up query processing (Moritz et al. 2017). Where possible, it can be useful to parallelise data processing and rendering so that they are performed simultaneously rather than sequentially (Hassan and Pernul 2014).

\subsection{Data Reduction}

As mentioned in Sect. 4.3, computer screens have a limited number of pixels onto which data points can be rendered. A possible solution is to make use of data reduction techniques to utilise the "screen real estate" efficiently (Yang et al. 2015). There are a number of ways to reduce the data, including filtering, clustering, and sampling techniques (Masiane 2019). Examples of sampling techniques that could be used to reduce the dataset include stratified random sampling, systematic random sampling, and quota sampling. Probability sampling techniques select the data in such a way that each data point has an equal probability of being selected. In contrast, non-probability sampling techniques are useful in some cases for the purposes of including at least one data point within each group or category that may be present in the data, meaning that data points which fall into a smaller group or category have a higher chance of being selected than data points which fall into larger categories or groups. This ensures each group is represented in the sample by the inclusion of at least one data point from each group or category (Genender-Feltheimer 2018).

An inevitable disadvantage of data reduction is information loss (Abidi et al. 2018) resulting in the inability to identify nuances such as outliers as well as the true patterns and trends in the data (Keck et al. 2017). 


\subsection{User Assistance}

Currently, the majority of visual analytics is carried out by domain experts, or mathematicians and statisticians with the appropriate analytical software and resources (Ko and Chang 2018). However, with increasingly user friendly web-based visualisation tools, many more novice users or laymen could conduct data analysis in future (Seokyeon et al. 2015). According to Behrisch et al., however, the visualisation tools that are commercially available currently still require much improvement in order to provide users with appropriate assistance (Behrisch et al. 2019). Examples of methods to provide the user with assistance include recommending which visualisation techniques are most suitable, given a particular dataset as input (Seokyeon et al. 2015). Behrisch et al. (2019) recommend calculating statistics as soon as the input data is loaded and displaying the results to the user immediately. The visualisation tool could further assist the user by predicting user queries based on use case, user profile, and the relevant information could be displayed based on these parameters.

Human Computer Interaction (HCI) principles are highly relevant in this domain and the user experience could be enhanced by incorporating principles such as gestault laws of grouping, and providing frequent and appropriate feedback (Seokyeon et al. 2015). Similarly, the use of colours is a simple way to highlight similarities or differences in the data (Elaiza et al. 2014). As mentioned in Sect. 4.2, visualisation tools must keep the user informed at each stage of the analysis to give the user situational awareness, inform the user of the progress of the query, and to give the user more control over the analysis (Behrisch et al. 2019; Zhao et al. 2017). Zhao et al. (2017) argue that most visual analytic systems are designed too specifically, thus they are only suitable for a limited range of situations as they restrict data types and data structures. A potential solution to make visualisation systems more dynamic and flexible is through customisation and the use of plug-ins and dynamically linked libraries (Liu 2019).

\section{Conclusion}

Big data and the visualisation of large datasets brings many promising opportunities for discoveries of patterns within the data. However, to fully capitalise on these opportunities, visualisation tools must be able to: perform dimensional reduction of multidimensional datasets; perform data reduction as it is not feasible to render each data point; ensure scalability and readability of visualisations; allow the user to interact with the data and visually analyse the data; retrieve results quickly so that the user does not lose their focus; and provide user assistance. Visualisation tools are currently too narrowly focused, and must be more flexible in terms of the data types they are able to process and the functions they are able to perform. As big data continually grows, data visualisation tools must improve rapidly to enable the exploration and analysis of the masses of data that are produced. Data visualization tools must improve at an equally rapid pace. 


\section{References}

Abidi, F., Polys, N., Rajamohan, S., Arsenault, L.: Remote high performance visualization of big data for immersive science (2018)

Agrawal, R., Dai, X., Andres, F.: Challenges and opportunities with big data visualization (2015)

Ali, A., Qadir, J., ur Rasool, R., Sathiaseelan, A., Zwitter, A.: Big data for development: applications and techniques. Big Data Anal. 1, 2 (2016). https://doi.org/10.1186/s41044-0160002-4

Behrisch, M., et al.: Commercial visual analytics systems - advances in the big data analytics field. IEEE Trans. Vis. Comput. Graph. 25(10), 3011-3031 (2019). https://doi.org/10.1109/ TVCG.2018.2859973

Bikakis, N.: Big data visualization tools, pp. 1-11 (2018)

Chen, Y., Guan, Z., Zhang, R., Du, X., Wang, Y.: A survey on visualization approaches for exploring association relationships in graph data. J. Vis. 22(3), 625-639 (2019). https://doi. org/10.1007/s12650-019-00551-y

Cho, W., Lee, H., Varma, M. K., Lee, M.: Big data analysis with interactive visualization using R packages (2014)

Elaiza, N., Khalid, A., Yusoff, M., Kamaru-zaman, E.A., Izzati, I.: Multidimensional data medical dataset using interactive visualization star coordinate technique. Proc. - Proc. Comput. Sci. 42, 247-254 (2014). https://doi.org/10.1016/j.procs.2014.11.059

Eldawy, A., Mokbel, M.F., Jonathan, C.: A demonstration of HadoopViz - an extensible MapReduce system for visualizing big spatial data, p 1896 (2015)

Elmqvist, N., Fekete, J.D.: Hierarchical aggregation for information visualization: overview, techniques, and design guidelines. IEEE Trans. Vis. Comput. Graph. 16(3), 439-454 (2010). https://doi.org/10.1109/TVCG.2009.84

Fernandez, A., Gonzalez, A., Diaz, J., Dorronsoro, J.: Diffusion maps for dimensionality reduction and visualization of meteorological data. Neurocomputing 163, 25-37 (2015)

Genender-Feltheimer, A.: Visualizing high dimensional and big data. In: Complex Adaptive Systems Conference with Theme: Cyber Physical Systems and Deep Learning. Chicago, Illinois, USA (2018)

Gisbrecht, A.: Advances in dissimilarity-based data visualisation. Bielefeld University (2013)

Gisbrecht, A., Hammer, B.: Data visualization by nonlinear dimensionality reduction. Wiley Interdisc. Rev.: Data Min. Knowl. Discov. 5(April), 51-74 (2015). https://doi.org/10.1002/ widm. 1147

Hassan, S., Pernul, G.: Efficiently managing the security and costs of big data storage using visual analytics categories and subject descriptors (2014)

Katal, A., Wazid, M., Goudar, R.H.: Big data: issues, challenges, tools and Good practices. In: 2013 6th International Conference on Contemporary Computing, IC3 2013, pp. 404-409 (2013). https://doi.org/10.1109/IC3.2013.6612229

Keck, M., et al.: Towards glyph-based visualizations for big data clustering. In: Proceedings of VINCI 2017, Bangkok, Thailand, 14-16 August 2017, p. 129 (2017)

Ko, I., Chang, H.: Interactive data visualization based on conventional statistical findings for antihypertensive prescriptions using national health insurance claims data. Int. J. Med. Inform. 116(May), 1-8 (2018). https://doi.org/10.1016/j.ijmedinf.2018.05.003

Li, X., Kuroda, A., Matsuzaki, H.: Polyspector ${ }^{\mathrm{TM}}$ : an interactive visualization platform optimized for visual analysis of big data, pp. 109-111 (2016)

Liu, Z.: Advances in engineering software a prototype framework for parallel visualization of large flow data. Adv. Eng. Softw. 130(December 2018), 14-23 (2019). https://doi.org/10. 1016/j.advengsoft.2019.02.004 
Van Long, T., Linsen, L.: Visualizing high density clusters in multidimensional data using optimized star coordinates. Comput. Stat. 26, 655-678 (2011). https://doi.org/10.1007/ s00180-011-0271-3

Lugmayr, A., Greenfeld, A., Zhang, D.J.: Selected advanced data visualizations : "the UXmachine", cultural visualisation, cognitive big data, and communication of health and wellness data, pp. 247-251 (2017)

Marr, B.: How much data do we create every day? The mind-blowing stats everyone should read (2018). https://www.forbes.com/sites/bernardmarr/2018/05/21/how-much-data-do-we-createevery-day-the-mind-blowing-stats-everyone-should-read/\#5d18565f60ba\%0Ahttps://www. forbes.com/sites/bernardmarr/2018/05/21/how-much-data-do-we-create-every-day-the-mindblowing-st. Accessed 31 Mar 2019

Masiane, M.: Towards insight driven sampling for big data visualisation (2019)

Molina-solana, M., Birch, D., Guo, Y.: Improving data exploration in graphs with fuzzy logic and large-scale visualisation. Appl. Soft Comput. J. 53, 227-235 (2017). https://doi.org/10. 1016/j.asoc.2016.12.044

Moritz, D., Fisher, D., Ding, B., Wang, C.: Trust but verify: optimistic visualizations of approximate queries for exploring big data, p. 2904 (2017)

Mwangi, B., Soares, J.C., Hasan, K.M.: Visualization and unsupervised predictive clustering of high-dimensional multimodal neuroimaging data. J. Neurosci. Methods 236, 1-7 (2014)

Noyes, D.: The top 20 valuable Facebook statistics - updated December 2015 (2019a).https:// zephoria.com/top-15-valuable-facebook-statistics/. Accessed 31 Mar 2019

Noyes, D.:. Top 10 Twitter Statistics - Updated March 2019 (2019b). https://zephoria.com/ twitter-statistics-top-ten/. Accessed 31 Mar 2019

Okoli, C., Schabram, K.: A guide to conducting a systematic literature review of information systems research. 10(2010) (n.d.)

Olshannikova, E., Ometov, A., Koucheryavy, Y., Olsson, T.: Visualizing big data with augmented and virtual reality: challenges and research agenda. J. Big Data 1-27 (2015). https://doi.org/10.1186/s40537-015-0031-2

Orad, A.: 2019 Gartner Magic Quadrant (2019). https://www.sisense.com/gartner-magicquadrant-business-intelligence/. Accessed 6 Jan 2020

Resnyansky, L.: Conceptual frameworks for social and cultural big data analytics: answering the epistemological challenge. Big Data Soc. 6(1), 205395171882381 (2019). https://doi.org/10. $1177 / 2053951718823815$

Ruan, G., Zhang, H.: Closed-loop big data analysis with visualization and scalable. Big Data Res. 8, 12-26 (2017). https://doi.org/10.1016/j.bdr.2017.01.002

Seokyeon, K., et al.: Big data visual analytics system for disease pattern analysis, p 175 (2015)

Shirota, Y., Hashimoto, T., Basabi, C.: Visualization challenge on time series statistical data, p. 12 (2017)

Sullivan, D.: Google now handles at least 2 trillion searches per year (2016). http:// searchengineland.com/google-now-handles-2-999-trillion-searches-per-year-250247. Accessed 31 Mar 2019

Wang, L., Wang, G., Alexander, C.A.: Big data and visualization: methods, challenges and technology progress. Digit. Technol. 1(1), 33-38 (2015). https://doi.org/10.12691/dt-1-1-7

Wang, S., Li, W.: Computers, environment and urban systems capturing the dance of the earth: polarglobe: real-time scientific visualization of vector field data to support climate science. Comput. Environ. Urban Syst. 77(June), 101352 (2019). https://doi.org/10.1016/j. compenvurbsys.2019.101352

Xie, Y., Chenna, P., Le, L., Planteen, J.: Visualization of big high dimensional data in three dimensional space. In: 3rd International Conference on Big Data Computing, Applications and Technologies (2016) 
Yang, Y., Zhang, K., Wang, J., Nguyen, Q.V.: Cabinet tree: an orthogonal enclosure approach to visualizing and exploring big data. J. Big Data 2, 15 (2015). https://doi.org/10.1186/s40537015-0022-3

Zhao, H., Zhang, H., Liu, Y., Zhang, Y., Luke, X.: Pattern discovery: a progressive visual analytic design to support categorical data analysis. J. Vis. Lang. Comput. 43, 42-49 (2017). https://doi.org/10.1016/j.jvlc.2017.05.004

Zhu, S.-C.: Statistical modeling and conceptualization of visual patterns. IEEE Trans. Pattern Anal. Mach. Intell. 25(6), 691-712 (2003). https://doi.org/10.1109/tpami.2003.1201820

Zou, Q., Zeng, J., Cao, L., Ji, R.: A novel features ranking metric with application to scalable visual and bioinformatics data classification. Neurocomputing 173, 346-354 (2016). https:// doi.org/10.1016/j.neucom.2014.12.123 